\title{
Sparse Frequency Waveform Design for Radar-Embedded Communication
}

\author{
Chaoyun Mai, ${ }^{1}$ Jinping Sun, ${ }^{1}$ Rui Zhou, ${ }^{1}$ and Guohua Wang ${ }^{2}$ \\ ${ }^{1}$ School of Electronic and Information Engineering, Beihang University, Beijing 100191, China \\ ${ }^{2}$ Temasek Laboratories, Nanyang Technological University, Singapore 117411
}

Correspondence should be addressed to Jinping Sun; sunjinping@buaa.edu.cn

Received 8 January 2016; Revised 11 May 2016; Accepted 26 May 2016

Academic Editor: Nazrul Islam

Copyright (c) 2016 Chaoyun Mai et al. This is an open access article distributed under the Creative Commons Attribution License, which permits unrestricted use, distribution, and reproduction in any medium, provided the original work is properly cited.

\begin{abstract}
According to the Tag application with function of covert communication, a method for sparse frequency waveform design based on radar-embedded communication is proposed. Firstly, sparse frequency waveforms are designed based on power spectral density fitting and quasi-Newton method. Secondly, the eigenvalue decomposition of the sparse frequency waveform sequence is used to get the dominant space. Finally the communication waveforms are designed through the projection of orthogonal pseudorandom vectors in the vertical subspace. Compared with the linear frequency modulation waveform, the sparse frequency waveform can further improve the bandwidth occupation of communication signals, thus achieving higher communication rate. A certain correlation exists between the reciprocally orthogonal communication signals samples and the sparse frequency waveform, which guarantees the low SER (signal error rate) and LPI (low probability of intercept). The simulation results verify the effectiveness of this method.
\end{abstract}

\section{Introduction}

The purpose of general radar-communication integration design is to achieve high bandwidth data communications between radar platforms by using radar transmitter/receiver subsystem. Different from former methods, Blunt proposed a method of intrapulse radar-embedded communication applied to the Tag [1], which is a new battlefield IFF (Identification Friend or Foe) equipment. The active communication capability between Tag and radar provides further information about the target area, for example, the exact location. Compared with the general radar-communication integration majoring in the sharing ability between radar waveform and communication signals, radar-embedded communication system focuses more on concealment of communication signals. The best way to avoid being detected by enemy devices near the Tag is to embed the Tag signal into radar echo [2]. The basic principle of radar-embedded communications is shown in Figure 1. The Tag device installed in the radar detection zone receives the radar signals, embeds the communication signals by remodulation, and transmits them as radar scattering echoes to radar receiver and achieves covert communication $[3,4]$. The communication signals usually choose orthogonal waveforms, which are widely used in radar waveform design.

Researches of intrapulse radar-embedded communications in [5-9] focus on linear frequency modulation (LFM) pulses. In [7, 8], Ciuonzo proposed a novel waveform design procedure based on multiobjective optimization. The framework of design strategies for intrapulse radar-embedded communications is summarized in [9]. Due to nearly flat spectral characteristic of LFM, the embedded communication signals can only utilize a small range of spectrum band, which leads to limited available communication waveform samples and low efficiency of communication. In view of this problem, a method for sparse frequency waveform design based on radar-embedded communication is proposed. It can improve the band utilization of the communication signal, increase the number of communication samples, and achieve low signal error rate and low probability of intercept while the detecting performance of the radar will not be degraded.

Sparse frequency waveform design is an important research direction of the radar waveform design. For the radar system, sparse frequency waveforms have advantages 


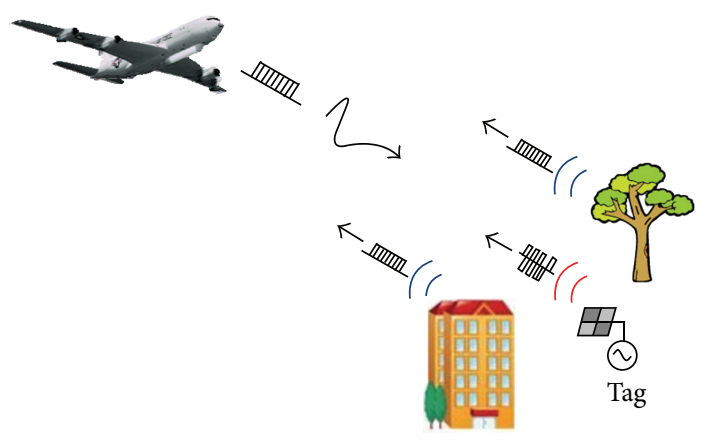

FIGURE 1: Radar-embedded communications applied to the Tag.

of suppressing interference and improving detection performance. To design a specific sparse frequency waveform, power spectral density fitting can be used to get the objective function [10-15] by using quasi-Newton method and then the eigenvalue decomposition of sparse frequency waveform sequence can be further utilized to obtain the dominant space. Following that $K$ communication waveforms are designed through the projection of orthogonal pseudorandom vectors in the nondominant subspace. Those $K$ communication waveform samples are orthogonal to each other, with strong independence, but have certain relevance with the sparse frequency waveforms. These features ensure that the information would not be easily intercepted by enemy radar during the communication process. Therefore, the use of sparse frequency waveform stopband can effectively increase the frequency band occupation of communication signals, to gain a higher communication rate.

\section{Radar-Embedded Communication Model}

The Tag device in detection range of pulse radar receives radar signals and embeds communication signals by remodulation. Then the output signals are mixed with echo of surrounding scene and return to the radar receiver. The received signals can be expressed as

$$
y_{r}(t)=\alpha_{k} c_{k}(t)+y_{s}(t)+n(t)
$$

where $n(t)$ describes system noise, $y_{s}(t)$ describes ambient radar echoes of the Tag device, $\alpha_{k}$ describes the combined effect of the path loss, $c_{k}(t)$ describes communication signals designed, $k=1,2, \ldots, K$ means the $k$ th communication waveform samples, and $K$ is the total number of samples. Rewrite formula (1) in vector form as

$$
\mathbf{y}_{r}=\alpha_{k} \mathbf{c}_{k}+\mathbf{S} \mathbf{x}+\mathbf{n}
$$

where $\boldsymbol{c}_{k}$ is the communication signals, $\mathbf{n}$ is the noise vector, $\mathbf{x}$ is the range samples of radar scattering around Tag device, and $\mathbf{S x}$ is discrete representation of convolution process between the radar waveform and the scattering response.
Given waveform vector $\mathbf{s}=\left[\begin{array}{llll}s_{0} & s_{1} & \cdots & s_{N-1}\end{array}\right]^{T}$, the Toeplitz matrix $\mathbf{S}$ can be expressed as

$$
\mathbf{S}=\left[\begin{array}{ccccccc}
s_{N-1} & s_{N-2} & \cdots & s_{0} & 0 & \cdots & 0 \\
0 & s_{N-1} & s_{N-2} & \cdots & s_{0} & \cdots & 0 \\
\cdots & \cdots & \cdots & \cdots & \cdots & \cdots & \cdots \\
0 & 0 & \cdots & s_{N-1} & s_{N-2} & \cdots & s_{0}
\end{array}\right]
$$

To ensure the concealment (low probability of intercept) of communication signals, the power of $c_{k}(t)$ must be lower than that of the echo signal $y_{s}(t)$. When there is a correlation between $y_{s}(t)$ and communication signal $c_{k}(t)$, it is difficult for standard coherent filter to isolate communication samples from the mixed signals. To solve this problem, a design of correlation receiver was proposed in [1].

As communication receiving is relatively simple (embedding only one of $K$ communication samples) and the relative power of the signal is unknown (decided by $\alpha_{k}$ ), a maximum likelihood receiver (known as decorrelator receiver) can be used to realize the communication receiving function. The length of sequence is set to $N$. The $N \times(2 N+K-1)$ matrix can be formed as

$$
\mathbf{C}=\left[\begin{array}{llll}
\mathbf{S} & \mathbf{c}_{1} & \cdots & \mathbf{c}_{K}
\end{array}\right] .
$$

Assuming that the noise is white Gaussian $\mathcal{N}\left(0, \sigma_{v}^{2}\right)$, the probability density function of the received signal $\mathbf{y}_{r}$ parameterized with $\mathbf{z}=\left[\begin{array}{llll}\mathbf{x}^{T} & \alpha_{1} & \cdots & \alpha_{K}\end{array}\right]$ can be expressed as

$$
p\left(\mathbf{y}_{r} ; \mathbf{z}\right)=\frac{1}{\left(2 \pi \sigma_{v}^{2}\right)^{N / 2}} \exp \left\{-\frac{\left(\mathbf{y}_{r}-\mathbf{C z}\right)^{H}\left(\mathbf{y}_{r}-\mathbf{C z}\right)}{2 \sigma_{v}^{2}}\right\} \text {. }
$$

The maximum likelihood estimate of parameter $\mathbf{z}$ can therefore be obtained:

$$
\widehat{\mathbf{z}}=(\mathbf{C C})^{-1} \mathbf{C}^{H} \mathbf{y}_{r} .
$$

For embedded communication, only the most likely one of the $K$ communication waveform samples is needed to be found. The decorrelator receiver [4] of the $k$ th sample can be expressed as

$$
\mathbf{w}_{k}=\left(\mathbf{C C}^{H}\right)^{-1} \mathbf{c}_{k}
$$

After obtaining $\mathbf{w}_{1}, \mathbf{w}_{2}, \ldots, \mathbf{w}_{K}$, correlate each of them with the received signal $\mathbf{y}_{r}$, respectively, and the maximum likelihood estimate of $k$ can be calculated by

$$
\widehat{k}=\arg \left\{\max _{k}\left\{\left|\mathbf{w}_{k}^{H} \mathbf{y}_{r}\right|\right\}\right\} .
$$

The maximum likelihood receiver of formula (7) is the minimum variance unbiased estimator of parameter $\mathbf{z}$ for the linear model in formula (2). And formula (8) is the minimum distance receiver for multiple hypothesis tests. 


\section{Sparse Frequency Waveform Design of Embedded Communications}

3.1. Sparse Frequency Radar Waveform Design. A sparse frequency waveform with specific passband and stopband could be obtained by power spectrum fitting. Given the distribution of power spectral density $\mathbf{u}$, the objective function of sparse frequency waveform can be expressed as

$$
\min _{\Theta}\left\|(\operatorname{As}(\Theta)) \otimes(\operatorname{As}(\Theta))^{*}-\mathbf{u}\right\|^{2},
$$

where s represents the designed waveform, whose phase vector $\Theta=\left[\phi_{1} \cdots \phi_{N}\right], \mathbf{A}$ is the discrete Fourier transform matrix.

The objective function (9) is a quartic nonconvex optimization problem. It can be solved by computing its local minimum rather than the global minimum with the quasiNewton method [10]. The quasi-Newton method uses information of the objective function value and its first derivative to construct the curvature approximation of the objective function, so it has the advantages of fast convergence. The gradient of the objective function needs to be calculated in each iteration.

The objective function (9) can be rewritten as

$$
\begin{aligned}
f(\Theta) & =\left\|(\mathbf{A s}(\Theta)) \otimes(\operatorname{As}(\Theta))^{*}-\mathbf{u}\right\|^{2} \\
& =\| \begin{array}{c}
\mathbf{a}_{1}^{H} \mathbf{s}(\Theta) \mathbf{s}^{H}(\Theta) \mathbf{a}_{1}-u_{1} \\
\vdots \\
\mathbf{a}_{N}^{H} \mathbf{s}(\Theta) \mathbf{s}^{H}(\Theta) \mathbf{a}_{N}-u_{N_{f}}
\end{array} \mid \\
& =\sum_{m=1}^{N}\left|\mathbf{s}^{H}(\Theta) \mathbf{a}_{m} \mathbf{a}_{m}^{H} \mathbf{s}^{H}(\Theta)-u_{m}\right|^{2} \\
& =\sum_{m=1}^{N}\left|\mathbf{s}^{H}(\Theta) \mathbf{A}_{m} \mathbf{s}^{H}(\Theta)-u_{m}\right|^{2} .
\end{aligned}
$$

The derivative with respect to $\Theta$ of the objective function can be expressed as

$$
\begin{aligned}
\mathbf{g}= & \frac{\partial f(\Theta)}{\partial \Theta} \\
= & 2 \sum_{m=1}^{N}\left(\mathbf{s}(\Theta) \mathbf{A}_{m} \mathbf{s}^{H}(\Theta)-u_{m}\right) \\
& \cdot 2 \operatorname{Im}\left\{\operatorname{diag}\{\mathbf{s}(\Theta)\} \mathbf{A}_{m}^{H} \mathbf{s}^{H}(\Theta)\right\} .
\end{aligned}
$$

Specific iterative steps of sparse frequency waveform design are as follows.

Step 1. Initialize $\Theta_{0}$ and tolerance $\varepsilon$, set $k=0$ and $\mathbf{S}_{0}=I$, and then calculate $\mathbf{g}_{0}$ by formula (11).

Step 2. Set the search direction $\mathbf{d}_{k}=-\mathbf{S}_{k} \mathbf{g}_{k}$, calculate $f\left(\Theta_{k}+\right.$ $\alpha_{k} \mathbf{d}_{k}$ ), and find the optimal step size $\alpha_{k}$ by line search method so that $f\left(\Theta_{k}+\alpha_{k} \mathbf{d}_{k}\right)$ is minimized; let $\boldsymbol{\delta}_{k}=\alpha_{k} \mathbf{d}_{k}$ and $\Theta_{k+1}=$ $\Theta_{k}+\boldsymbol{\delta}_{k}$.
Step 3. If $\left\|\boldsymbol{\delta}_{k}\right\| \leq \varepsilon$, end the iteration and output $\Theta_{\text {optim }}=$ $\Theta_{k+1}$; if $\left\|\boldsymbol{\delta}_{k}\right\|>\varepsilon$, go to Step 4 .

Step 4. Calculate $\mathbf{g}_{k+1}$, set $\boldsymbol{\gamma}_{k}=\mathbf{g}_{k+1}-\mathbf{g}_{k}$, and then compute

$$
\begin{gathered}
\mathbf{S}_{k+1}=\mathbf{S}_{k}+\left(1+\frac{\boldsymbol{\gamma}_{k}^{H} \mathbf{S}_{k} \boldsymbol{\gamma}_{k}}{\boldsymbol{\gamma}_{k}^{H} \boldsymbol{\delta}_{k}}\right) \frac{\boldsymbol{\delta}_{k} \boldsymbol{\delta}_{k}^{H}}{\boldsymbol{\gamma}_{k}^{H} \boldsymbol{\delta}_{k}} \\
-\frac{\boldsymbol{\delta}_{k} \boldsymbol{\gamma}_{k}^{H} \mathbf{S}_{k}+\mathbf{S}_{k} \boldsymbol{\gamma}_{k} \boldsymbol{\delta}_{k}^{H}}{\boldsymbol{\gamma}_{k}^{H} \boldsymbol{\delta}_{k}} .
\end{gathered}
$$

Set $k=k+1$ and repeat from Step 2 .

Set an appropriate value of $\varepsilon$ (e.g., $10^{-3}$ ); then the sparse frequency radar waveform with a distribution close to that of the ideal power spectral density can be obtained by the quasiNewton method described above.

3.2. Waveform Design of Embedded Communications. First of all, the eigenvalue decomposition of the sparse frequency waveform sequence designed is used to get the dominant space. Then $K$ communication waveform samples are designed through the projection of orthogonal pseudorandom vectors in the nondominant subspace [4].

Set the length of sparse frequency radar waveform sequence $N$. Known from formulas (1) and (2), radar scattering echo around the Tag within the radar illumination region can be discretely represented as

$$
\mathbf{y}_{s}=\mathbf{S} \mathbf{x}
$$

where $\mathbf{S}$ is a $N \times(2 N-1)$ Toeplitz matrix. $\mathbf{x}$ is a $2 N-1$ vector, representing range samples of radar scattering.

After the eigenvalue decomposition of $\mathbf{S}, N$ eigenvalues $\lambda_{0}, \lambda_{1}, \ldots, \lambda_{N-1}$ and corresponding eigenvectors $\mathbf{v}_{0}, \mathbf{v}_{1}$, $\ldots, \mathbf{v}_{N-1}$ can be calculated. With eigenvalues in ascending order (i.e., $\lambda_{0} \leq \lambda_{1} \leq \cdots \leq \lambda_{N-1}$ ), constitute the matrix $\mathbf{V}=\left[\begin{array}{llll}\mathbf{v}_{0} & \mathbf{v}_{1} & \cdots & \mathbf{v}_{N-1}\end{array}\right]$ accordingly. The eigenvalue decomposition is shown as

$$
\mathbf{S S}^{H}=\mathbf{V} \boldsymbol{\Lambda} \mathbf{V}^{H}
$$

where $\Lambda$ is a diagonal matrix which contains $N$ associated eigenvalues.

A sequence will be obtained if the eigenvalues are sorted in ascending order and it clearly exhibits a demarcation that the large eigenvalues in the sequence correspond to the passband while small eigenvalues correspond to the stopband. Assuming that the space spanned by eigenvectors corresponding to the first $L$ eigenvalues is the nondominant space, as $\operatorname{span}\left\{\mathbf{V}_{o}\right\}=\operatorname{span}\left\{\mathbf{v}_{0}, \mathbf{v}_{1}, \ldots, \mathbf{v}_{L-1}\right\}$, the space spanned by eigenvectors corresponding to the remaining $N-L$ eigenvalues is the dominant space, as $\operatorname{span}\left\{\mathbf{V}_{s}\right\}=$ $\operatorname{span}\left\{\mathbf{v}_{L}, \mathbf{v}_{L+1}, \ldots, \mathbf{v}_{N-1}\right\}$. The frequency spectrum of the stopband should be fully utilized to avoid the effect on the performance when designing communication waveform samples. The design steps are as follows.

First of all, generate a set of $K$ pseudorandom $N$ dimension vectors denoted by $\mathbf{b}_{k}, k=1,2, \ldots, K$ (both Tag 
and receiver know the specific values of pseudorandom vectors $\mathbf{b}_{k}$ ). Then by projecting the orthogonal pseudorandom vector $\mathbf{b}_{k}$ to its nondominant subspace, according to [4], the first communication sample $\mathbf{c}_{1}$ can be designed as

$$
\mathbf{c}_{1}=\left(\mathbf{I}-\mathbf{V}_{s, 0} \mathbf{V}_{s, 0}^{H}\right) \mathbf{b}_{1},
$$

where $\mathbf{V}_{s, 0}=\left[\begin{array}{llll}\mathbf{v}_{L} & \mathbf{v}_{L+1} & \cdots & \mathbf{v}_{N-1}\end{array}\right]$. Matrices $\mathbf{S}$ and $\mathbf{c}_{1}$ are combined to obtain a $N \times 2 N$ matrix $\mathbf{S}_{1}=\left[\begin{array}{ll}\mathbf{S} & \mathbf{c}_{1}\end{array}\right]$. Do the eigenvalue decomposition again and $N$ eigenvalues $\lambda_{0}^{\prime}, \lambda_{1}^{\prime}, \ldots, \lambda_{N-1}^{\prime}$ of $\mathbf{S}_{1}$ will be obtained as well as corresponding eigenvectors $\mathbf{v}_{0}^{\prime}, \mathbf{v}_{1}^{\prime}, \ldots, \mathbf{v}_{N-1}^{\prime}$. The eigenvalue decomposition is shown as

$$
\mathbf{S}_{1} \mathbf{S}_{1}^{H}=\mathbf{V}_{1} \Lambda_{1} \mathbf{V}_{1}^{H},
$$

where $\mathbf{V}_{1}=\left[\begin{array}{llll}\mathbf{v}_{0}^{\prime} & \mathbf{v}_{1}^{\prime} & \cdots & \mathbf{v}_{N-1}^{\prime}\end{array}\right]$ and $\boldsymbol{\Lambda}_{1}$ is a diagonal matrix which contains $N$ associated eigenvalues. The nondominant space of $\mathbf{V}_{1}$ is $\operatorname{span}\left\{\mathbf{V}_{o, 1}\right\}=\operatorname{span}\left\{\mathbf{v}_{0}^{\prime}, \mathbf{v}_{1}^{\prime}, \ldots, \mathbf{v}_{L-2}^{\prime}\right\}$ and the dominant space of $\mathbf{V}_{1}$ is $\operatorname{span}\left\{\mathbf{V}_{s, 1}\right\}=$ $\operatorname{span}\left\{\mathbf{v}_{L-1}^{\prime}, \mathbf{v}_{L}^{\prime}, \ldots, \mathbf{v}_{N-1}^{\prime}\right\}$.

Then the second communication sample can be designed as

$$
\mathbf{c}_{2}=\left(\mathbf{I}-\mathbf{V}_{s, 1} \mathbf{V}_{s, 1}^{H}\right) \mathbf{b}_{2},
$$

where $\mathbf{V}_{s, 1}=\left[\begin{array}{llll}\mathbf{v}_{L-1}^{\prime} & \mathbf{v}_{L}^{\prime} & \cdots & \mathbf{v}_{N-1}^{\prime}\end{array}\right]$. Thus, matrices $\mathbf{S}$ and $\mathbf{c}_{1}, \mathbf{c}_{2}, \ldots, \mathbf{c}_{k-1}$ can be combined to obtain an $N \times(2 N+$ $k-1)$ matrix $\mathbf{S}_{k-1}=\left[\begin{array}{lllll}\mathbf{S} & \mathbf{c}_{1} & \mathbf{c}_{2} & \cdots & \mathbf{c}_{k-1}\end{array}\right] . N$ eigenvalues $\lambda_{0}^{(k-1)}, \lambda_{1}^{(k-1)}, \ldots, \lambda_{N-1}^{(k-1)}$ and corresponding eigenvectors $\mathbf{v}_{0}^{(k-1)}, \mathbf{v}_{1}^{(k-1)}, \ldots, \mathbf{v}_{N-1}^{(k-1)}$ of $\mathbf{S}_{k-1}$ can be further obtained after the eigenvalue decomposition shown as

$$
\mathbf{S}_{k-1} \mathbf{S}_{k-1}^{H}=\mathbf{V}_{k-1} \mathbf{\Lambda}_{k-1} \mathbf{V}_{k-1}^{H} \text {, }
$$

where $\mathbf{V}_{k-1}=\left[\begin{array}{llll}\mathbf{v}_{0}^{(k-1)} & \mathbf{v}_{1}^{(k-1)} & \ldots & \mathbf{v}_{N-1}^{(k-1)}\end{array}\right]$ and $\boldsymbol{\Lambda}_{1}$ is a diagonal matrix which contains $N$ associated eigenvalues. The nondominant space of $\mathbf{V}_{k-1}$ is $\operatorname{span}\left\{\mathbf{V}_{o, k-1}\right\}=$ $\operatorname{span}\left\{\mathbf{v}_{0}^{(k-1)}, \mathbf{v}_{1}^{(k-1)}, \ldots, \mathbf{v}_{L-k}^{(k-1)}\right\}$ and the dominant space of $\mathbf{V}_{k-1}$ is $\operatorname{span}\left\{\mathbf{V}_{o, k-1}\right\}=\operatorname{span}\left\{\mathbf{v}_{0}^{(k-1)}, \mathbf{v}_{1}^{(k-1)}, \ldots, \mathbf{v}_{L-k}^{(k-1)}\right\}$. Then the $k$ th communication sample can be designed as

$$
\mathbf{c}_{k}=\left(\mathbf{I}-\mathbf{V}_{s, k-1} \mathbf{V}_{s, k-1}^{H}\right) \mathbf{b}_{k},
$$

where $\mathbf{V}_{s, k-1}=\left[\begin{array}{llll}\mathbf{v}_{L-k+1}^{(k-1)} & \mathbf{v}_{L-k+2}^{(k-1)} & \cdots & \mathbf{v}_{N-1}^{(k-1)}\end{array}\right]$

An important indicator of covert communication is interception [3]. Since the conventional method based on measuring the spectral energy is no longer applicable, Blunt et al. proposed the normalized correlation to measure interception [4]. The intended radar waveform is assumed to be known by the enemy intercept receiver, and the dominant space of waveform is obtained through the eigenvalue decomposition by formula (14). The $N \times l$ matrix $\widetilde{\mathbf{V}}_{s, l}$ is formed by the eigenvectors corresponding to the largest $l$ eigenvalues. The projection of received signal in the nondominant subspace of the main space can be considered as nonradar echo component. The $l$ th projected value of the received signal $\mathbf{y}_{r}$ is

$$
\mathbf{a}_{l}=\mathbf{P}_{l} \mathbf{y}_{r}
$$

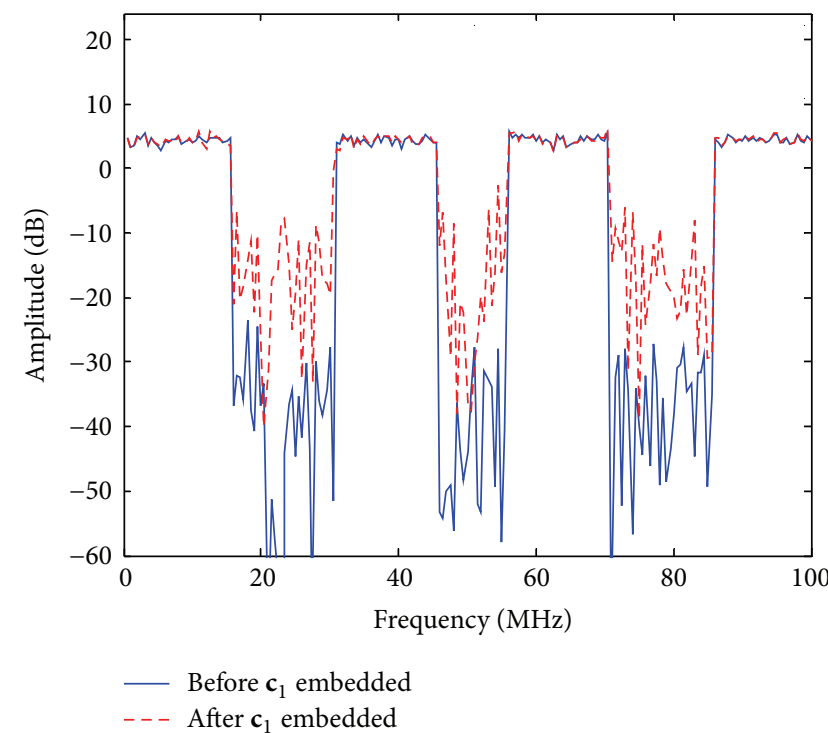

Figure 2: Power spectral density of radar waveform after communication signal $\mathbf{c}_{1}$ embedded.

where $\mathbf{P}_{l}=\mathbf{I}-\tilde{\mathbf{V}}_{s, l} \tilde{\mathbf{V}}_{s, l}^{H}, l \in[1, \ldots, N]$ is the projection matrix corresponding to different $l$.

Then, normalized correlation of the $k$ th communication waveform is defined as

$$
\eta_{k, l}=\frac{\left|\mathbf{c}_{k}^{H} \mathbf{a}_{l}\right|}{\sqrt{\left(\mathbf{c}_{k}^{H} \mathbf{c}_{k}\right)\left(\mathbf{a}_{l}^{H} \mathbf{a}_{l}\right)}} .
$$

$\eta_{k, l}$, having a range of ( $\left.\begin{array}{ll}0 & 1\end{array}\right)$, can be used to measure the intercept probability of communications waveforms [4]. The larger value of $\eta_{k, l}$ indicates higher similarity between the communication signal detected by the intercept receiver and the actual embedded signal, which means higher probability to be intercepted. Communication waveforms are thought to have no covertness when $\eta_{k, l}$ approaches 1 .

\section{Experimental Results}

Set the length of the sparse frequency radar waveform sequence $N=200$. The desired power spectral density u has 4 passbands and 3 stopbands. The set of passbands is $5 \mathrm{db}$ and stopbands $-30 \mathrm{~dB}$. $\varepsilon$ is presumed to be 0.001 . According to the iterative steps from Section 3.1, the obtained power spectral density of sparse frequency waveform is shown in Figure 2.

As the sparse frequency waveforms have been obtained, communication waveforms can be designed through the method discussed in Section 3.2. Set the total number of communication samples $K=16$ and compute $\mathbf{c}_{1}, \mathbf{c}_{2}, \ldots, \mathbf{c}_{16}$. Figure 2 shows the power spectral density of radar waveform after the communication signal $\mathbf{c}_{1}$ is embedded; that leads to the increased power in the stopband obviously. We can also see the change for the radar waveform in time domain when the communication signal $\mathbf{c}_{1}$ is embedded. The result waveforms in time domain before and after $\mathbf{c}_{1}$ embedded are shown in Figure 3. 


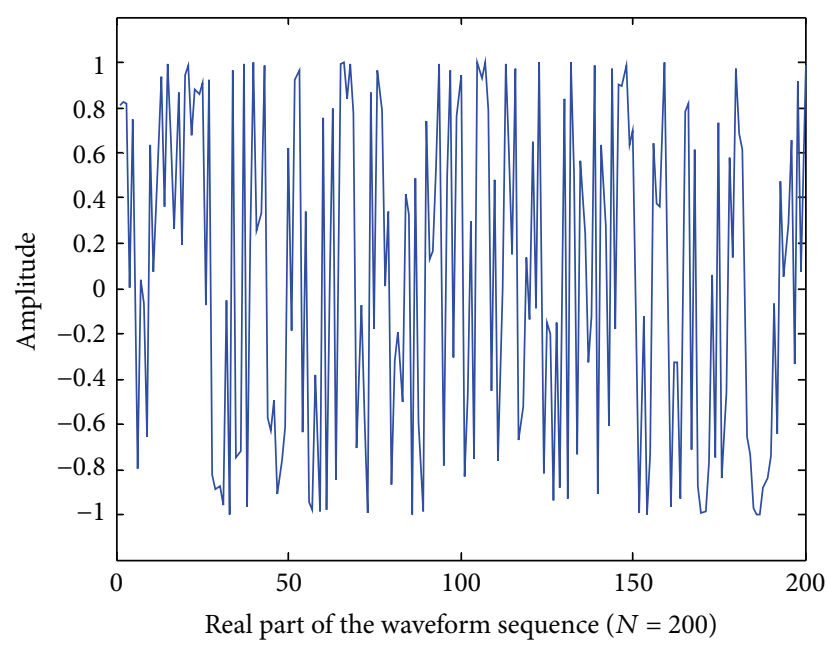

(a)

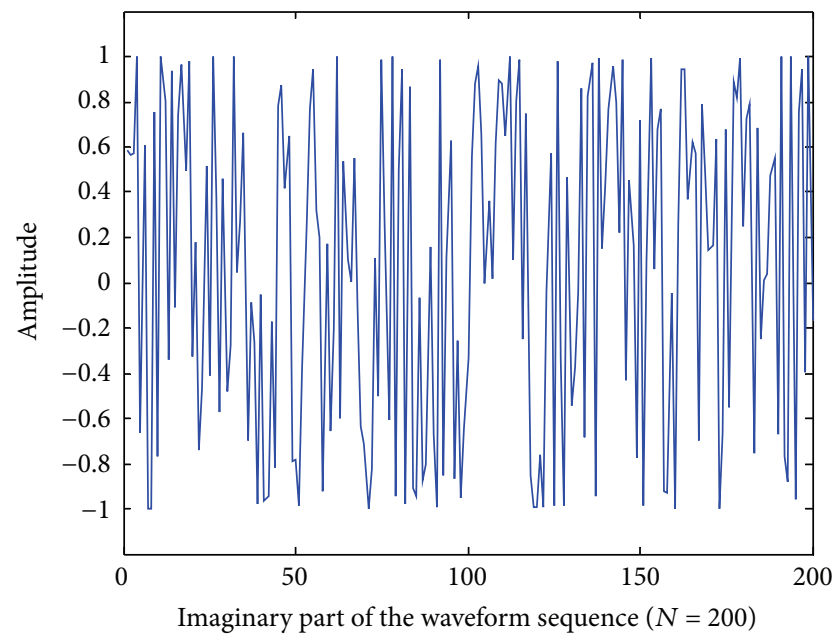

(c)

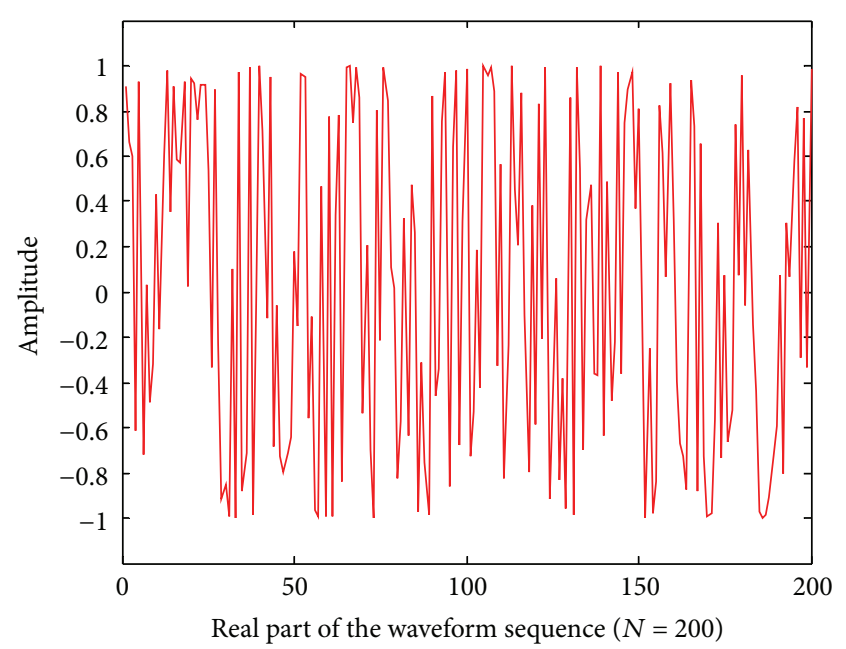

(b)

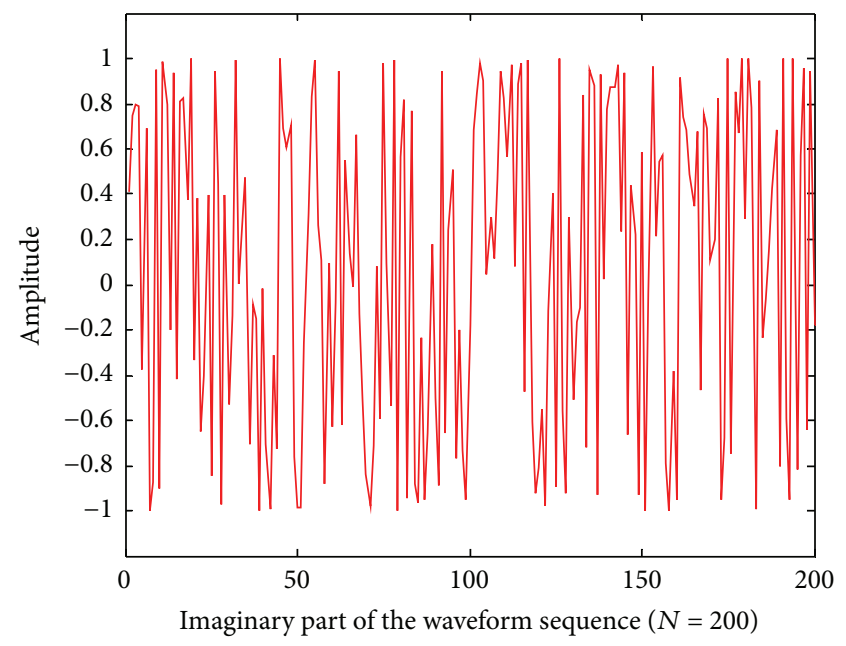

(d)

FIGURE 3: Sparse frequency radar waveform in time domain. (a) The real part before $\mathbf{c}_{1}$ embedded; (b) the real part after $\mathbf{c}_{1}$ embedded; (c) the imaginary part before $\mathbf{c}_{1}$ embedded; (d) the imaginary part after $\mathbf{c}_{1}$ embedded.

Figures 3(a) and 3(c) show the real part and the imaginary part of the original sparse frequency radar waveform, which is a multiphase coded waveform. It is constant modulus, and the modulus value is normalized. Figures $3(b)$ and $3(d)$ show the real part and the imaginary part of the waveform after the communication signal $\mathbf{c}_{1}$ is embedded, and it can be seen that the difference is not obvious in time domain. For the case that $\mathbf{c}_{2}-\mathbf{c}_{16}$ embedded, the results are similar.

After obtaining the radar-embedded communication waveforms, we can further analyze the SER with the variation of SNR. This design method contains $K=16$ kinds of communication waveforms and the first one as an example is adopted in simulation (the probability is evaluated when the intended receiver successfully detects the communication signal $\mathbf{c}_{1}$ transmitted by transmitter). Change the power of noise to vary the value of SNR from $-25 \mathrm{~dB}$ to $0 \mathrm{~dB}$. Set the number of nondominant eigenvectors used by Tag and radar receiver $L=80$, and the number of large eigenvalues used by intercept receiver $j=120$. The result averaged over 10000 Monte Carlo simulations is shown in Figure 4.

As is shown, when the SNR is $-8 \mathrm{~dB}$, SER can achieve $10^{-3}$ by using a decorrelator receiver, far less than that of the intercept receiver. It demonstrates that the communication waveform has good concealment. Figure 4 only shows the result of $\mathbf{c}_{1}$. The results of samples of $\mathbf{c}_{2}-\mathbf{c}_{16}$ are similar to that of $c_{1}$. When SNR ranges from $-10 \mathrm{~dB}$ to $-5 \mathrm{~dB}$, the decorrelator receiver has lower SER.

The interception of communication waveforms is further analyzed. Set the SNR to $-15 \mathrm{~dB}$. While calculating the normalized correlation, the number of large eigenvalues of the intercept receiver $l$ varies from 0 to 200 (i.e., change from $0-100 \%$, because $N=200$ ). Each point uses 200 samples to calculate $\eta_{k, 1}$ and get the average. Results are shown in Figure 5.

In Figure 5, the horizontal axis represents the percentage of the number of eigenvalues $l$; the vertical axis represents 


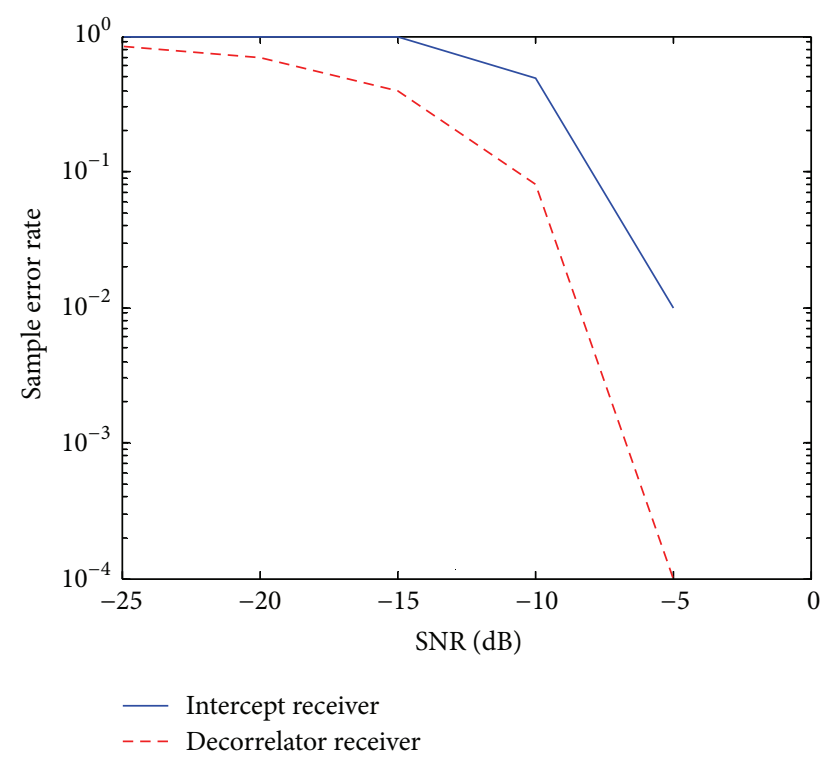

FIGURE 4: Error rate after communication signal $\mathbf{c}_{1}$ embedded.

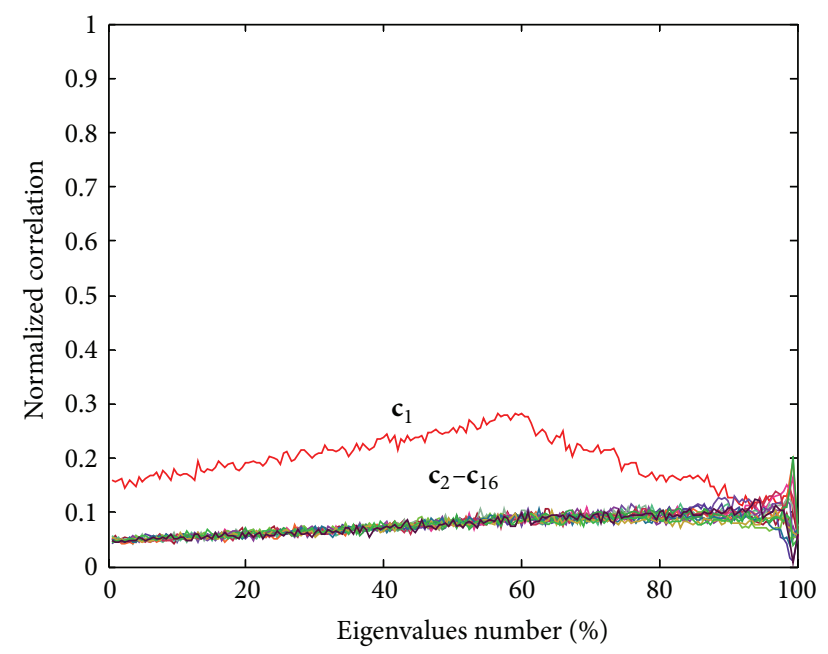

FIgURE 5: Normalized correlation curve by computation of waveform $c_{1}$.

the value of $\eta_{k, l}$. According to formula (20), the calculation of normalized correlation needs to know one of the communication waveforms. Supposing the communication sample $c_{1}$ is known, compute the normalized correlation of $\mathbf{c}_{2}, \mathbf{c}_{3}, \ldots, \mathbf{c}_{16}$ and also that of $\mathbf{c}_{1}$, for generality. It can be seen from Figure 5 that the maximum of $\eta_{k, l}$ corresponding to $\mathbf{c}_{2}-\mathbf{c}_{16}$ is less than 0.2 and the maximum of $\eta_{k, l}$ corresponding to $\mathbf{c}_{1}$ is approximately equal to 0.3 . Even increasing the percentage of eigenvalues, the waveform that is designed by this method can still maintain a good ability of interception. Simulation in Figure 5 only shows the result provided that the communication sample $\mathbf{c}_{1}$ is known. In the actual operation, using the communication samples $\mathbf{c}_{2}-\mathbf{c}_{16}$ will get the similar curve and the same conclusion.

The impact of embedded communication signals into radar signal by autocorrelation function is further analyzed. Figure 6 shows normalized autocorrelation function (ACF) of

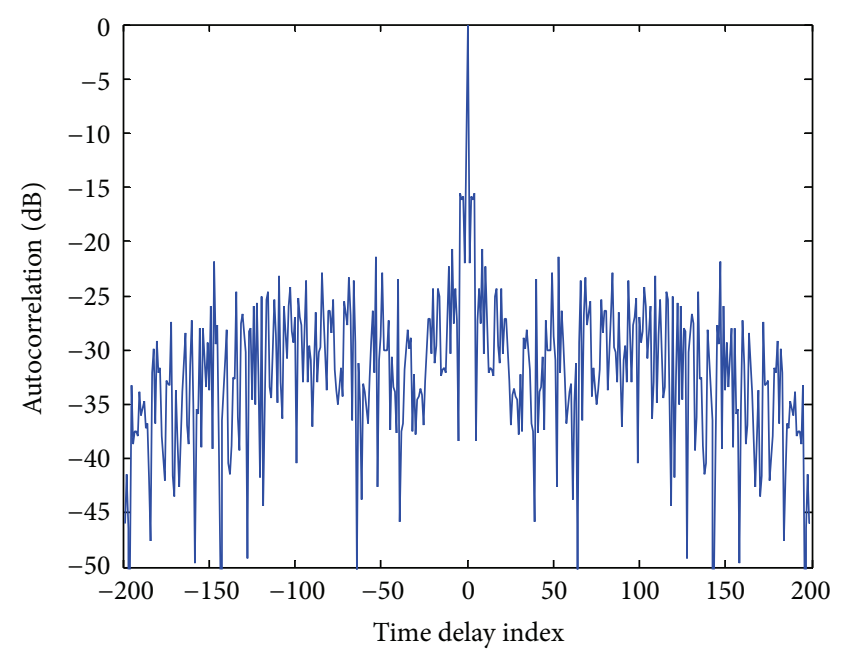

(a)

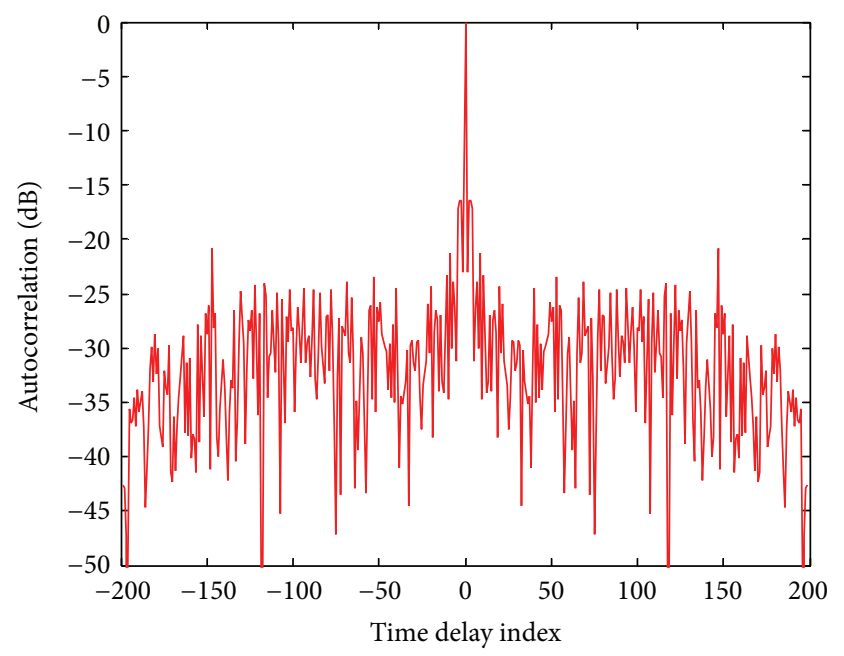

(b)

FIGURE 6: ACF of sparse frequency radar-embedded communication waveform. (a) Before $\mathbf{c}_{1}$ embedded; (b) after $\mathbf{c}_{1}$ embedded.

radar waveform [16] before and after the waveform embedded communication signals, with ordinate in logarithm form. Figure 6(a) shows the ACF under original sparse frequency radar waveform, and Figure 6(b) shows the ACF after adding the communication waveform, which would be $\mathbf{c}_{1}$. The integral sidelobe level (ISL) is $-10.19 \mathrm{~dB}$ and peak sidelobe level (PSL) is $-15.62 \mathrm{~dB}$ before communication waveform is embedded. And the ISL is $-10.94 \mathrm{~dB}$ and PSL is $-16.44 \mathrm{~dB}$ when communication waveform is embedded.

It can be seen that the autocorrelation performances are similar before or after the communication waveform $\mathbf{c}_{1}$ is embedded, which means the embedded communication waveforms do not affect the detection performance of the radar system. For $\mathbf{c}_{2}-\mathbf{c}_{16}$, the results are basically the same.

\section{Conclusions}

In this paper, we propose the sparse frequency waveform design based on radar-embedded communication to improve 
the performance of the existing radar Tag system with the function of covert communication. Firstly, sparse frequency waveforms are designed based on power spectral density fitting with the quasi-Newton method and the eigenvalue decomposition is used to get the dominant space of the waveform sequence. Secondly, the communication waveforms are designed through the projection of orthogonal pseudorandom vectors in the nondominant subspace. In the end, we analyze the error rate and the interception of sparse frequency waveform in which communication waveform samples are embedded under different SNR conditions in simulation. The result shows that using sparse frequency waveform can improve the bandwidth occupation of communication signals. Thus, a higher communication rate can be gained. Furthermore, these samples are orthogonal to each other, which guarantees low SER and LPI. It is also verified that the autocorrelation performance of radar signals would not be degraded by embedding communication signals.

\section{Competing Interests}

The authors declare that they have no competing interests.

\section{Acknowledgments}

This work was supported by the National Natural Science Foundation of China (Grant no. 61471019).

\section{References}

[1] S. D. Blunt and P. Yantham, "Waveform design for radarembedded communications," in Proceedings of the International Conference on Waveform Diversity and Design (WDD '07), pp. 214-218, Pisa, Italy, June 2007.

[2] D. Hounam and K.-H. Wägel, "A Technique for the identification and localization of sar targets using encoding transponders," IEEE Transactions on Geoscience and Remote Sensing, vol. 39, no. 1, pp. 3-7, 2001.

[3] S. D. Blunt, J. Stiles, C. Allen, D. Deavours, and E. Perrins, "Diversity aspects of radar-embedded communications," in Proceedings of the International Conference on Electromagnetics in Advanced Applications (ICEAA '07), pp. 439-442, Torino, Italy, September 2007.

[4] S. D. Blunt, P. Yatham, and J. Stiles, "Intrapulse radar-embedded communications," IEEE Transactions on Aerospace and Electronic Systems, vol. 46, no. 3, pp. 1185-1200, 2010.

[5] S. D. Blunt and C. R. Biggs, "Practical considerations for intrapulse radar-embedded communications," in Proceedings of the International Waveform Diversity and Design Conference (WDD '09), pp. 244-248, Kissimmee, Fla, USA, February 2009.

[6] S. D. Blunt and J. G. Metcalf, "Estimating temporal multipath via spatial selectivity: building environmental knowledge into waveform design for radar-embedded communications," in Proceedings of the International Conference on Electromagnetics in Advanced Applications (ICEAA '09), pp. 513-516, Torino, Italy, September 2009.

[7] D. Ciuonzo, A. De Maio, G. Foglia, and M. Piezzo, "Paretotheory for enabling covert intrapulse radar-embedded communications," in Proceedings of the IEEE Radar Conference
(RadarCon '15), pp. 292-297, IEEE, Arlington, Va, USA, May 2015.

[8] D. Ciuonzo, A. De Maio, G. Foglia, and M. Piezzo, "Intrapulse radar-embedded communications via multiobjective optimization," IEEE Transactions on Aerospace and Electronic Systems, vol. 51, no. 4, pp. 2960-2974, 2015.

[9] J. G. Metcalf, C. Sahin, S. D. Blunt, and M. Rangaswamy, "Analysis of symbol-design strategies for intrapulse radarembedded communications," IEEE Transactions on Aerospace and Electronic Systems, vol. 51, no. 4, pp. 2914-2931, 2015.

[10] G. H. Wang and Y. L. Lu, "Sparse frequency waveform design based on PSD fitting," in Proceedings of the 36th IEEE International Conference on Acoustics, Speech, and Signal Processing (ICASSP '11), pp. 2860-2863, Prague, Czech Republic, May 2011.

[11] G. H. Wang and Y. L. Lu, "Sparse frequency transmit waveform design with soft power constraint by using PSO algorithm," in Proceedings of the IEEE Radar Conference (RADAR '08), pp. 1-4, Rome, Italy, May 2008.

[12] G. H. Wang and Y. L. Lu, "Designing sparse frequency waveform using iterative algorithm," in Proceedings of the 11th International Radar Symposium (IRS '10), pp. 1-4, Vilnius, Lithuania, June 2010.

[13] G. H. Wang, C. Y. Mai, J. P. Sun, and Y. L. Lu, "Sparse frequency waveform analysis and design based on ambiguity function theory," IET Radar, Sonar \& Navigation, vol. 10, no. 4, pp. 707$717,2016$.

[14] G. H. Wang and Y. L. Lu, "Designing single/multiple sparse frequency waveforms with sidelobe constraint," IET Radar, Sonar and Navigation, vol. 5, no. 1, pp. 32-38, 2011.

[15] C. Y. Mai, S. T. Lu, J. P. Sun, and G. H. Wang, "Codesign of beam pattern and sparse frequency waveforms for MIMO radar," International Journal of Antennas and Propagation, vol. 2015, Article ID 563941, 12 pages, 2015.

[16] N. Levanon and E. Mozeson, Radar Signals, Wiley-IEEE Press, New York, NY, USA, 2004. 


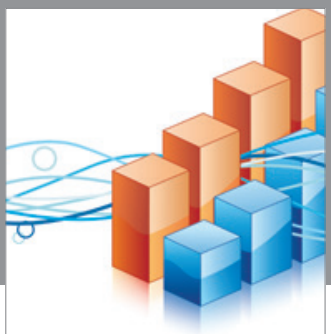

Advances in

Operations Research

vatem alat4

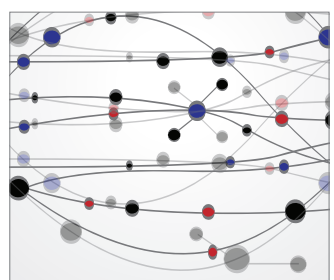

\section{The Scientific} World Journal
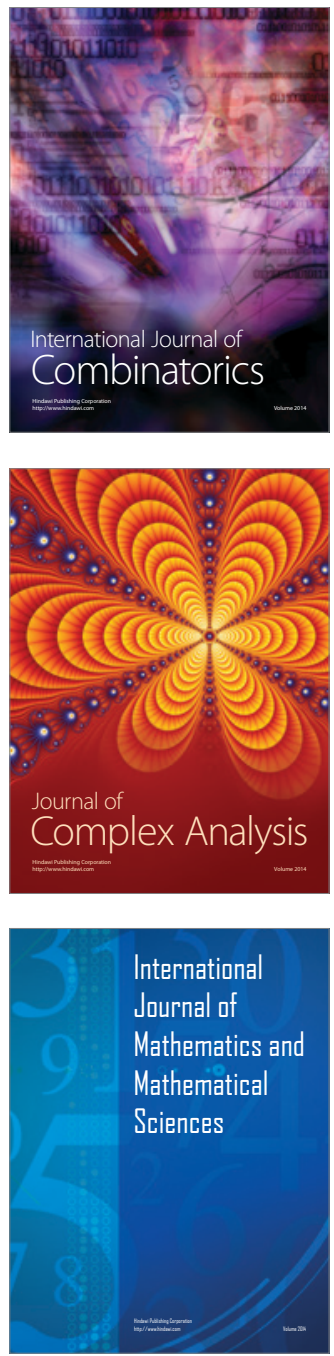
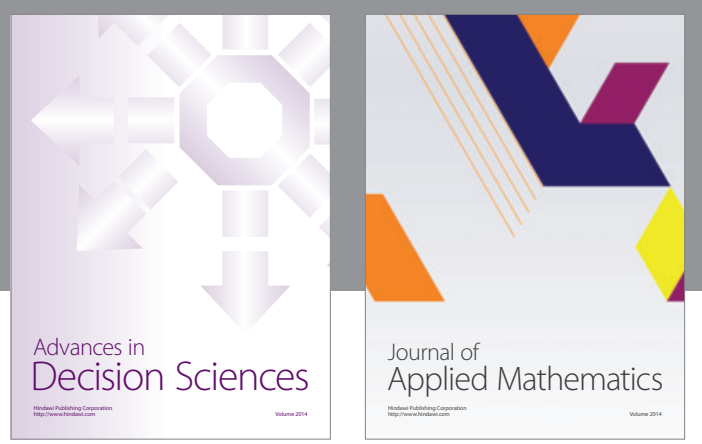

Algebra

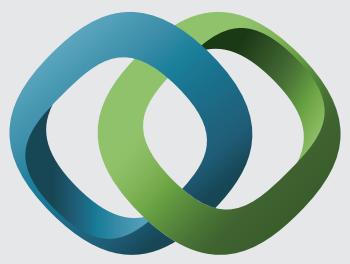

\section{Hindawi}

Submit your manuscripts at

http://www.hindawi.com
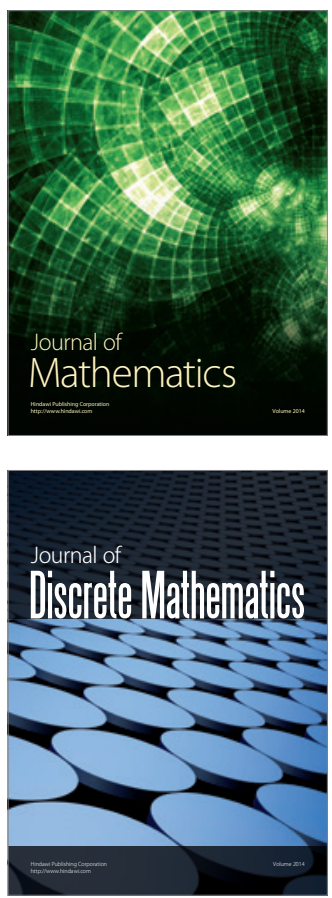

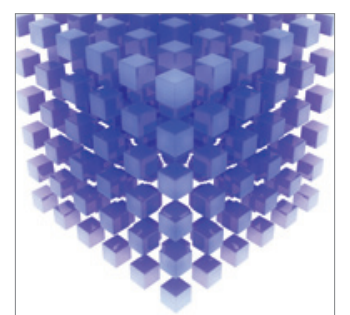

Mathematical Problems in Engineering
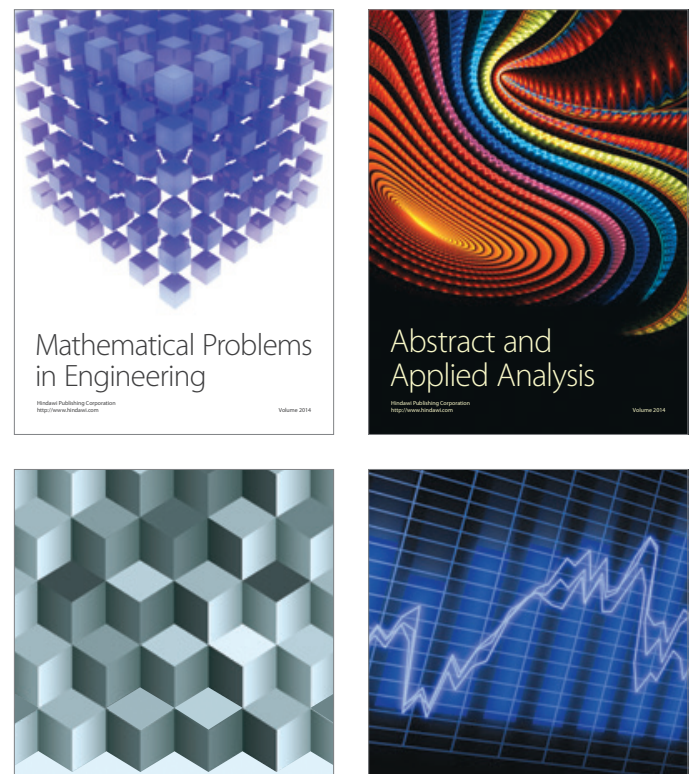

Journal of

Function Spaces

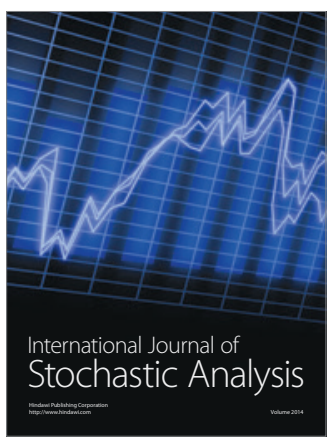

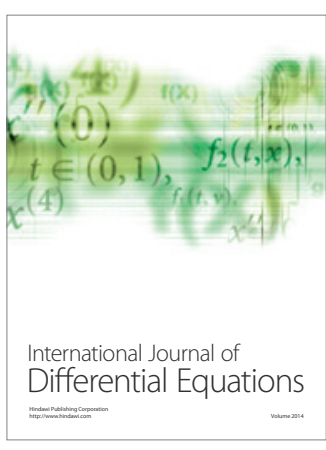
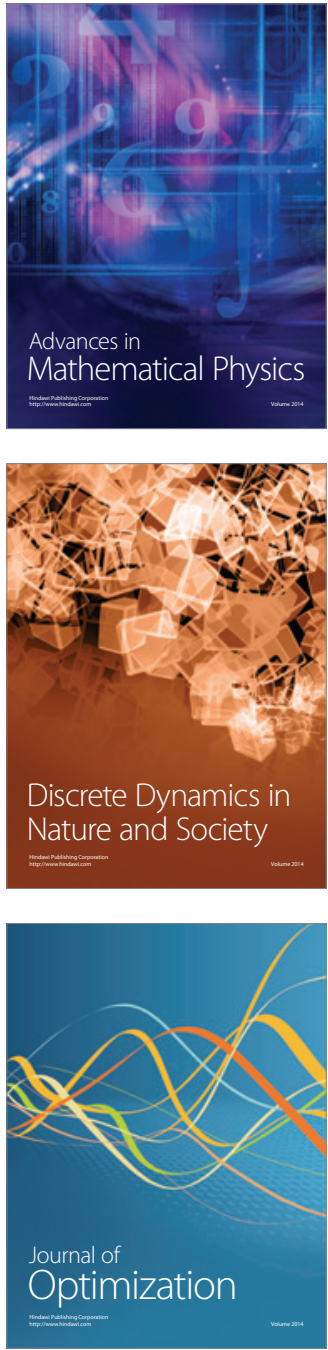